\title{
Estudo Morfométrico do Miocárdio em Adultos com Subnutrição Protéico-Energética
}

\author{
Daniel Ferreira da Cunha, Cristiane Helena Pedrini, Júlio Cláudio Sousa, Marlene Antônia dos Reis, \\ Sidney Gonçalves Ramos, Selma Freire de Carvalho da Cunha, Vicente de Paula Antunes Teixeira
}

Uberaba, MG

\begin{abstract}
Objetivo - Comparar o tamanho das células musculares e a presença de lipofuscina no coração de adultos necropsiados, subnutridos e controles.

Métodos - De uma casuística inicial de 315 necropsias de adultos, foram excluidos casos com sinais de retenção hídrica, cardiopatias, hepatopatias, hipertensão e nefropatias. Subnutrição foi caracterizada pelo índice de massa corporal (IMC) $<17 \mathrm{~kg} / \mathrm{m}^{2}$. Fragmentos de miocárdio foram processados para morfometria computadorizada, determinando-se a espessura dos miocardiócitos e a presença de lipofuscina.

Resultados - Subnutridos $(n=8)$ e controles $(n=4)$ foram estatisticamente diferentes, respectivamente, quanto ao $\operatorname{IMC}\left(14,86 \pm 1,13\right.$ vs $\left.22,02 \pm 0,9 \mathrm{~kg} / \mathrm{m}^{2}\right)$, relação peso cardíaco/peso corporal $(0,68 \pm 0,09$ vs $0,54 \pm 0,07 \%)$, espessura de miocardiócitos $(10,91 \pm 0,77 v s$ 12,90 $\pm 1,82 \mu \mathrm{m})$ e presença de lipofuscina no miocárdio (39,1 vs 54,4\%).

Conclusão-Comparados aos controles, adultos subnutridos têm menores espessuras de miocardiócitos e presença de lipofuscina, fenômenos que refletiriam um distúrbio metabólico com repercussões clínicas danosas em pacientes terminais.
\end{abstract}

Palavras-chaves: subnutrição protéico-energética, morfometria miocárdica, lipofuscina

\section{Myocardial Morphometry of Protein-Energy Malnourished Adults}

Purpose - To compare cardiac muscle cells width and cardiomyocyte lipofuscin pigment presence between malnourished and non-malnourished necropsied adults.

Methods - Out of 315 necropsy protocols of adults randomly chosen, those with edema, ascitis, systemic arterial hypertension, chronic liver disease, and heart disease were excluded. Malnutrition was defined by body mass index $(B M I)<17 \mathrm{~kg} / \mathrm{m}^{2}$. Cardiomyocytes morphometry study and lipofuscin pigment counts were performed.

Results-Malnourished $(n=8)$ and controls $(n=4)$, respectively, showed statistical differences in relation to

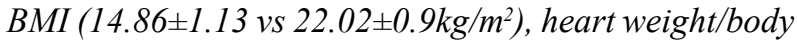
weight ratio ( $0.68 \pm 0.09$ vs $0.54 \pm 0.07 \%)$, cardiomyocytes width $(10.91 \pm 0.77 \mathrm{vs} 12.90 \pm 1.82 \mu \mathrm{m})$ and lipofuscin pigment presence (39.1 vs $54.4 \%)$.

Conclusion - When compared with controls, necropsied malnourished adults showed decreased myocardial fibers diameters and lower lipofuscin pigment presence. These findings might reflect altered metabolism, and would be associated with harmful clinical effects in terminally ill patients.

Key-words: protein-energy malnutrition, myocardium morphometry, lipofuscin

Arq Bras Cardiol, volume 71 (n 5), 677-680, 1998

A subnutrição protéico-energética (SPE) crônica, do tipo marasmo, decorre do aporte alimentar insuficiente para suprir as necessidades energéticas e protéicas do indivíduo, processo que se acompanha de emagrecimento pro-

Faculdade de Medicina do Triângulo Mineiro (FMTM) - Uberaba

Correspondência: Daniel Ferreira da Cunha - Faculdade de Medicina do Triângulo Mineiro - Depto de Clínica Médica - Nutrologia - Av. Getúlio Guaritá, S/N 38025-440 - Uberaba, MG

Recebido para publicação em 7/5/98

Aceito em 5/8/98 gressivo, com diminuição do peso corporal e das reservas de gordura e de massa muscular. Em adultos, a SPE do tipo kwashiorkor está associada com hipoalbuminemia e edema, geralmente com evolução mais rápida e muitas vezes desencadeada por infecção, trauma ou cirurgia ${ }^{1}$. Nos seres humanos, uma conseqüência da subnutrição é a progressiva perda das reservas corporais de musculatura e de tecido adiposo, com hipotrofia de diversos órgãos, incluindo rins, intestinos e órgãos linfóides ${ }^{1}$. O fígado (esteatose hepática) e os pulmões (maior susceptibilidade às infecções) também podem ser afetados ${ }^{2}$. 
Concepções teleológicas sobre a função cardíaca deram suporte à opinião, corrente entre os médicos, de que o coração seria preservado na subnutrição ${ }^{3}$. Entretanto, estudos com animais e necropsias de crianças com kwashiorkor mostraram diminuição da massa cardíaca, proporcional ao grau de emagrecimento. À microscopia de luz, as células cardíacas apresentam-se atrofiadas, com vacuolização e eventual fragmentação de miofibrilas ${ }^{4,5}$. Nas décadas de 70 e 80, foram descritos casos de morte súbita associada com arritmias cardíacas e síndrome do QTc longo em adultos obesos submetidos a dietas líquidas hipocalóricas, não balanceadas do ponto de vista eletrolítico. À microscopia, os miocardiócitos apresentavam-se atrofiados e circundados por intenso edema intersticial ${ }^{4,6}$.

Existem poucos estudos morfológicos do miocárdio de adultos subnutridos, possivelmente porque a SPE seja menos reconhecida e documentada neste grupo etário. Nestes casos, pode-se também quantificar a presença de lipofuscina, pigmento derivado da peroxidação lipídica de membranas subcelulares, que se acumula em neurônios e no coração de adultos, idosos ou subnutridos ${ }^{7,8}$.

O objetivo do presente estudo foi comparar o tamanho de células e núcleos de células musculares cardíacas de adultos necropsiados, com ou sem subnutrição protéicoenergética, bem como quantificar a presença de lipofuscina.

\section{Métodos}

O trabalho foi realizado nas Disciplinas de Patologia Geral e de Nutrologia da FMTM, após aprovação da Comissão de Ética em Pesquisa da Instituição. Numa primeira etapa, de um total de 315 necropsias, realizadas de dezembro/ 86 a janeiro/97, foram selecionados 100 protocolos de necropsias completas de adultos com idade $>20$ anos.

Os diagnósticos principais à necropsia e os dados gerais dos indivíduos, incluindo cor, sexo, idade, peso e altura foram registrados num banco de dados eletrônico. Posteriormente, foram excluídos 88 casos, devido à presença de ascite, edema, insuficiência cardíaca congestiva (ICC) (cardiomegalia associada com edema generalizado, derrame pleural e hepatomegalia), hepatopatia crônica (icterícia mais cirrose, hepatite virótica ou alcoólica), ou glomerulopatia com sinais de síndrome nefrótica. Outros critérios de exclusão foram a positividade das reações sorológicas para doença de Chagas e a presença de nefrosclerose genuína, situação associada à hipertensão arterial sistêmica. Indivíduos com insuficiência cardíaca não foram incluídos, para se evitar eventual superposição de quadros de caquexia cardíaca, onde as alterações metabólicas decorrem da resposta do indivíduo às citocinas ${ }^{9}$.

O estado nutricional foi definido pelo índice de massa corporal (IMC, $\mathrm{kg} / \mathrm{m}^{2}$ ), sendo considerados subnutridos os indivíduos com IMC $<17 \mathrm{~kg} / \mathrm{m}^{2}$, valor menor que o geralmente utilizado como critério de subnutrição ${ }^{10}$. Os controles tinham IMC $>22,5$ e $<27 \mathrm{~kg} / \mathrm{m}^{2}$, faixa de variação de pessoas normais, não-obesas ${ }^{10,11}$. A relação peso cardíaco $(\mathrm{g}) /$ peso corporal $(\mathrm{g})$ x 100, utilizada na definição de hipertrofia cardíaca, foi calculada em todos os $\operatorname{casos}^{12}$.
Fragmentos transmurais do terço médio da parede livre do ventrículo esquerdo foram retirados, fixados em formol a $10 \%$ e processados conforme rotina do laboratório de anatomia patológica, obtendo-se cortes com $6 \mu \mathrm{m}$, corados pela técnica de hematoxilina-eosina.

As medidas morfométricas dos miocardiócitos foram realizadas utilizando-se uma câmara de vídeo acoplada a um microscópio de luz comum, de onde as imagens eram transmitidas a um monitor integrado a uma mesa de medições gráficas. A mesa dispunha de um cursor móvel e estava ligada a um sistema computadorizado para análise de imagem (MOP-Videoplan ${ }^{\circledR}$ ).

A espessura do miocardiócito foi determinada na posição central da célula, em correspondência com a localização nuclear. Tanto as medidas dos núcleos quanto a espessura das células foram realizadas em cortes longitudinais do miocárdio. Utilizou-se o método da média acumulada para determinação do número de células a serem avaliadas, sendo medidas as espessuras de 30 miocardiócitos escolhidos ao acaso, bem como a área e o diâmetro dos respectivos núcleos. A presença de lipofuscina, investigada com um microscópio de luz comum com aumento final de 500X, foi considerada positiva quando os grânulos do pigmento, de aparência mais grosseira, eram visibilizados com nitidez no citoplasma, especialmente em localização perinuclear. Os exames morfométricos foram realizados sem que os observadores tivessem conhecimento do estado nutricional do indivíduo.

Após verificação da normalidade estatística dos dados obtidos, os grupos subnutridos e controles foram comparados usando-se o teste " $t$ " de Student (variáveis contínuas) e o teste do $\chi^{2}$ ou teste exato de Fisher (proporções). Foram consideradas significativas as diferenças comp $<0,05$.

\section{Resultados}

Foram selecionados 12 casos, sendo oito com subnutrição protéico-energética e quatro controles. Entre subnutridos e controles não houve diferença estatística, respectivamente, quanto à idade ( $33,6 \pm 12$ vs $42,7 \pm 7,3$ anos), proporção de sexo masculino:feminino (7:1 vs 3:1) e cor branca:não-branca (3:1 vs 1:1). Os principais diagnósticos à necropsia foram similares entre subnutridos e controles, respectivamente: broncopneumonia (75 vs 37,5\%), neoplasias ( $25 v s 25 \%$ ), síndrome da imunodeficiência adquirida (SIDA) ( $25 v s 50 \%$ ) e aterosclerose de aorta ( $25 v s$ $12,5 \%$ ). Nenhum paciente com SIDA mostrou sinais microscópicos de comprometimento cardíaco por degeneração, necrose, infiltrado inflamatório ou microorganismos oportunísticos.

As alturas dos indivíduos subnutridos $(1,65 \pm 0,14 \mathrm{~m}) \mathrm{e}$ controles $(1,63 \pm 0,15 \mathrm{~m})$ também foram similares, mas os subnutridos foram estatisticamente menos pesados $(41,0 \pm 7,4 \mathrm{vs}$ $59,5 \pm 11,7 \mathrm{~kg})$ e apresentaram menor IMC $(14,86 \pm 1,13$ vs $\left.22,02 \pm 0,9 \mathrm{~kg} / \mathrm{m}^{2}\right)$. O peso cardíaco não foi estatisticamente diferente entre subnutridos $(276,2 \pm 36,1 \mathrm{~g})$ e controles 


\begin{tabular}{|lc|}
\hline \multicolumn{1}{|c|}{ Tabela I - Medidas do núcleo e do citoplasma de miocardiócitos de indivíduos controles e subnutridos } \\
\hline Medidas & $\begin{array}{c}\text { Controles } \\
\text { (média } \pm \text { dp) }\end{array}$ \\
\hline Diâmetro nuclear máximo $(\mu \mathrm{m})$ & $\begin{array}{c}\text { Subnutridos } \\
(\text { média } \pm \text { dp) }\end{array}$ \\
Diâmetro nuclear mínimo $(\mu \mathrm{m})$ & $10,94 \pm 3,27$ \\
Área nuclear $\left(\mu \mathrm{m}^{2}\right)$ & $5,81 \pm 1,61$ \\
Espessura celular $(\mu \mathrm{m}) *$ & $46,04 \pm 7,30$ \\
\hline$* \mathrm{p}<0,01$. & $12,90 \pm 1,82$ \\
\hline
\end{tabular}

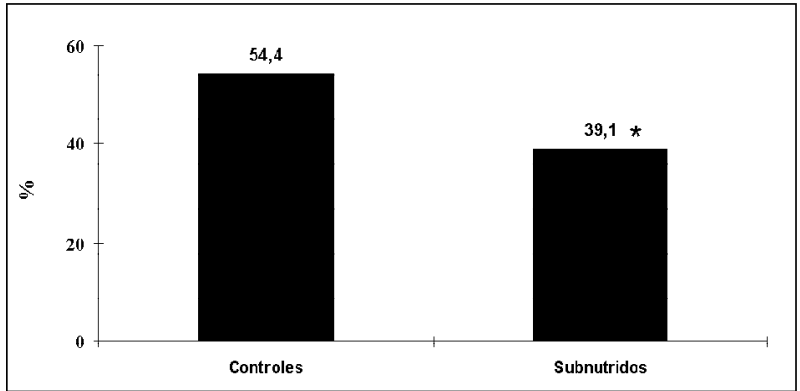

Fig. 1 - Freqüência de positividade de pigmento de lipofuscina em miocardiócitos de indivíduos adultos necropsiados, controles ou subnutridos. * $\mathrm{p}<0,05$.

$(322,5 \pm 76,76 \mathrm{~g})$. A relação peso cardíaco/peso corporal foi estatisticamente maior nos subnutridos $(0,68 \pm 0,09 \%)$, quando comparados aos controles $(0,54 \pm 0,07 \%)$.

As medidas dos diâmetros das células musculares e respectivos núcleos, bem como a área dos núcleos dos miocardiócitos de controles e subnutridos estão apresentadas na tabela I. A espessura das fibras miocárdicas foi estatisticamente menor no grupo subnutridos $(10,91 \pm 0,77 \mu \mathrm{m})$, quando comparada aos controles $(12,90 \pm 1,82 \mu \mathrm{m})$. Na figura 1 , observa-se a maior positividade de pigmentos de lipofuscina em miocardiócitos de casos controles $(54,4 \%)$ em comparação com os subnutridos $(39,1 \%)$.

\section{Discussão}

No presente trabalho, a utilização de um critério restritivo de subnutrição (IMC $<17 \mathrm{~kg} / \mathrm{m}^{2}$ ) e a exclusão de indivíduos obesos, com edema ou com valores intermediários de IMC, asseguram a distinção dos grupos subnutrido e controle. Da mesma forma, a exclusão de cardiopatas ou de portadores de doenças sistêmicas diminui a possibilidade de eventual presença de condições causadoras de hipertrofia miocárdica.

Cães submetidos a subnutrição experimental apresentam redução da espessura da parede dos ventrículos e do débito cardíaco, proporcionais à diminuição da massa corporal magra ${ }^{3}$. Tal fato não está de acordo com a maior relação peso cardíaco/peso corporal observada em indivíduos subnutridos, que sugere relativa preservação do peso cardíaco em relação à intensidade do emagrecimento corporal. A menor espessura dos miocardiócitos de indivíduos subnutridos está de acordo com a hipotrofia miofibrilar observada em animais, causada pela diminuição da síntese de componentes das miofibrilas ${ }^{13} \mathrm{e}$ aumento do catabolismo protéico miofibrilar, secundário ao aumento da atividade das proteinases lisossômicas ${ }^{14}$. A similitude das dimensões nucleares de subnutridos e controles sugerem também a manutenção do potencial recuperativo do coração. Em conjunto, estes achados sugerem que, na adaptação cardíaca à subnutrição grave, ocorreria hipotrofia de miocardiócitos associada com preservação de outros componentes estruturais, como o aumento do tecido conjuntivo e da densidade capilar ${ }^{15}$.

Observou-se menor presença de lipofuscina no coração de indivíduos subnutridos, dados que não correspondem à noção de que a deposição deste pigmento seja mais intensa quando o grau de catabolismo supera o anabolismo celular, como ocorre no envelhecimento ${ }^{7,8}$. Entretanto, sabe-se que a restrição de aporte energético, exemplificada pela inanição prolongada, reduz a geração de radicais livres, retardando o processo de envelhecimento ${ }^{16}$. Dessa forma, animais com aporte energético reduzido teriam menor acúmulo de lipofuscina, provavelmente em conseqüência da manutenção das atividades da aldeído-desidrogenase e da glutationa-S-transferase musculares ${ }^{14}$. Assim, embora haja deposição miocárdica de lipofuscina nas fases iniciais da subnutrição, pode-se especular que, devido à diminuição do metabolismo celular, este pigmento depositar-se-ia menos nas fases mais avançadas. Além disso, não se pode descartar a possibilidade da oxidação e consumo de componentes lipídicos e protéicos da lipofuscina, para geração de energia.

No presente trabalho, documentou-se menor espessura de miocardiócitos em indivíduos com subnutrição de grau avançado, fenômeno que talvez reflita o predomínio do catabolismo protéico sobre os processos anabólicos ${ }^{13}$. Estas alterações morfo-funcionais miocárdicas poderiam se manifestar clinicamente como ICC e arritmias cardíacas ${ }^{17}$, intercorrências comuns em pacientes terminais. 


\section{Referências}

1. McMahon MM, Bistrian BR - The physiology of nutritional assessment and therapy in protein-calorie malnutrition. Dis Mon 1990; 36: 373-417.

2. Kelsen SG - The effects of undernutrition on the respiratory muscles. Clin Chest Med 1986; 7: 101-10

3. Alden PB, Madoff RD, Stahl TJ, Lakatua DJ, Ring WS, Cerra FB - Left ventricular function in malnutrition. Am J Physiol 1987; 253: H380-7.

4. Webb JG, Kiess MC, Chan-Yan CC - Malnutrition and the heart. Can Med Assoc J 1986; 135: 753-8.

5. Vandewoude MF, Buyssens $\mathrm{N}$ - Effect of ageing and malnutrition on rat myocardium. I. The myocyte. Virchows Arch A Pathol Anat Histopathol 1992; 421: 179-88.

6. Donnelly JE, Jakicic J, Gunderson S - Diet and body composition. Effect of very low calorie diets and exercise. Sports Med 1991; 12: 237-49.

7. Katz ML - Incomplete proteolysis may contribute to lipofuscin accumulation in the retinal pigment epithelium. Adv Exp Med Biol 1989; 266: 109-16

8. Wolf G - Lipofuscin, the age pigment. Nutr Rev 1993; 51: 205-6.

9. Freeman LM, Roubenoff R - The nutrition implications of cardiac cachexia. Nutr Rev 1994; 52: 340-7.

10. Heymsfield SB, Tighe A, Wang Z - Nutritional assessment by anthropometric and biomedical methods. In: Shils ME, Olson JA, Shike M, eds - Modern Nutrition in Health and Disease. $8^{\text {th }}$ ed. Philadelphia: Lea \& Febiger, 1994: 812-41.
11. James WP, Ferro-Luzzi A, Waterlow JC - Definition of chronic energy deficiency in adults. Report of a working party of the International Dietary Energy Consultative Group. Eur J Clin Nutr 1988; 42: 969-81.

12. Almeida HO, Teixeira VPA, Araújo WF-Comportamento do peso do coração e do corpo em chagásicos crônicos com e sem "megas". Rev Soc Bras Med Trop 1979/ 1980: 13: 85-9.

13. Samarel AM, Parmacek MS, Magid NM, Decker RS, Lesch M - Protein synthesi and degradation during starvation-induced cardiac atrophy in rabbits. Circ Res 1987; 60: 933-41.

14. Wildenthal K, Poole AR, Glauert AM, Dingle JT - Dietary control of cardiac lysosomal enzyme activities. Recent Adv Stud Cardiac Struct Metab 1975; 8: 519-29.

15. Vandewoude MF - Morphometric changes in microvasculature in rat myocardium during malnutrition. J Parenter Enteral Nutr 1995; 19: 376-80.

16. Masoro EJ - Retardation of aging processes by food restriction: an experimental tool. Am J Clin Nutr 1992; 55: S1250-2.

17. Cunha DF, Ferreira TPS, Almeida MA, Vergara ML, Cunha SFC-Correlation of nutritional status and QTc interval in electrocardiogram (EKG) of HIV (+) patients. In: I Conference on Nutrition and HIV Infection. Cannes - França, 1995: 57 